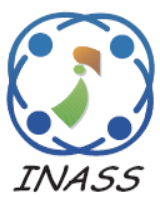

\title{
Enhancement of an Algorithm for Oil Tank Detection in Satellite Images
}

\author{
Niketan Janakraj Jivane ${ }^{1 *}$ \\ Rajkumar Soundrapandiyan ${ }^{1}$ \\ ${ }^{I}$ School of Computer Science \& Engineering, Vellore Institute of Technology, Vellore, India \\ * Corresponding author's Email: nike18tan@gmail.com
}

\begin{abstract}
Satellite imaging is the order of the day. Automatic oil tank detection is one of the important domains in satellite image processing which could be used for disaster monitoring, oil leakage, etc. It has great significance for the military and civilian application to detect oil tanks and locate oil depots automatically from remote satellite images. Automatic oil tank detection in satellite image remains a challenging problem. This paper addresses this problem using enhancement of algorithm for oil tank detection with SURF technique and SVM classifier approach. The proposed approach consists of four stages are pre-processing, segmentation, feature extraction and classification. Initially, the input image is pre-processed using threshold method. Secondly, SURF technique applied on the preprocessed image for the purpose of segment the oil tank. Then, well known features are extracted from the segmented image. Finally, to classify the oil tank and non oil tank support vector machine (SVM) is applied on the extracted feature. To test the proposed method the experiment is carried out the standard benchmark databases are SPOT-5 satellite images, QuickBird satellite images, GeoEye-1 satellite images and Google Earth images. The classification results obtained from the SVM classifier shows the efficiency of proposed method by high oil tank detection rate and low false alarm rate.
\end{abstract}

Keywords: SURF, Histogram oriented gradient, Feature extraction, Oil tank detection, SVM classifier.

\section{Introduction}

With the success of remote sensing technology, more high-resolution data are now available, facilitating a wide range of applications such as city surveying, disaster monitoring, and military reconnaissance. In these applications, automatic oil tank detection plays a major role. In industrial facilities to store oil and/or petrochemical products are described as oil depots. The primary structure of an oil depot is the tankage, either above ground or underground, wherein such valuable products are stored. One of the key imperatives is the safety of industrial facilities. The experiences expose that these regions are highly vulnerable, especially to natural disasters. The devastating instance occurred in Sendai, Japan caused massive damages to the region once the oil depots of the largest refinery in Japan were set ablaze by the earthquake. Therefore, risk evaluation of such regions prior to natural disasters is crucial, and could be performed with the help of remotely sensed images. Aerial/satellite images could be useful for locating individual oil tanks, providing information about their content. This may eventually help services responsible for the emergency planning, rescuing operations, and protecting individuals in the nearby residential areas.

Many previous studies in this context assume that oil depots are bright features, whose foreground is clearly separable from the neighbouring background, which eventually simplifies the problem. However, these studies do not evaluate their approaches for difficult conditions (e.g. complex background, varying seasonal effects such as illumination, shadow, smoke, and snow cover). Conversely, such cases are quite common for aerial/satellite images; thus, reduces the applicability and generalization of the previously developed approaches.

In this paper, we propose approach to automatically detect circular objects from high resolution satellite images. The approach considers the symmetric nature of the circular oil depots and 
allows us to detect them even in difficult conditions. The proposed approach consists of four steps are pre-processing, segmentation, feature extraction and classification. In first step, the input image is preprocessed using threshold method. In second, SURF technique is used for the purpose of segment the oil tank. In third step, well known features such as Histogram Oriented Gradient (HOG), Gray Level Co-occurrence matrix (GLCM), Completed Local Binary Pattern (CLBP), Centre Symmetric Local Binary Pattern (CSLBP), unique Unsorted vec are extracted from the segmented image. Finally, to classify the oil tank and non oil tank support vector machine (SVM) is applied on the extracted feature. Experimental results show that proposed approach can effectively detect oil tank on large-field, highresolution optical images and achieved promising results in precise. The advantages of the proposed method are as follows:

No need of any prior information about input image.

- Adaptive calculations of parameters required for oil tank classification.

- The proposed method handles any size of images.

- The proposed method efficiently detects the oil tanks.

- The proposed method yields high detection rate and low false rate.

The rest of the paper is organized as follows: Section 2 presents the literature survey. Section 3 briefly discussed about proposed methodology. Preprocessing is discussed in Section 3.1, Candidate detection is presented in Section 3.2, feature extraction is presented in Section 3.3 and classification is discussed in Section 3.4. Experimental results and performance analysis are presented in Section 4. Section 5 concludes the paper.

\section{Literature survey}

L. Zhang et al [1] proposed method which used the ELSD (Ellipse \& Line segment Detector) for candidate selection, HOG (Histogram of oriented gradient) to detect local area, CNN (Convolution Neural network) to detect surrounding area SVM classifier to confirm tank. There are some issues in this method are i) This method is sensitive to different resolutions, ii) Lower resolution could result in more missing targets, iii) The targets near the image borders may not include enough surrounding area and may give a wrong decision result because of the poor representation of the surrounding features, iv) It focuses on local information than surrounding information and sometimes surrounding image is has lot information than local image, and v) Detection accuracy could be even lower if they cannot reliably grasp the surrounding information. They miss some targets in the meantime as the complex surrounding information needs a higher requirement for the feature. Here, Google earth benchmark database used for testing.

N. Dalal et al [2] took databases from MIT pedestrian database and INRIA. They used Reminiscent of edge orientation histogram, SIFT descriptor and Shape context methods for detecting target. There some drawbacks which are i) Gradients should be calculated at the finest available scale for human detection both wavelets and shape contexts lose out significantly here, ii) Strong local contrast normalization is essential for good results, and traditional centre-surround style schemes are not the best choice, iii) Better results can be achieved by normalizing each element (edge, cell) several times with respect to different local supports, and treating the results as independent signals, iv) There is still room for optimization and to further speed up detections it would be useful to develop a coarse-tofine or rejection chain style detector based on HOG descriptors and v) Failed to incorporate motion information using block matching or optical flow fields.

A. O. Ok et al [3] presented the automated thresholding method. In this method, computation of gradient are applied on images and then applying 5tap coefficient method for detecting oil depots. It failed to process oblique images. There is no validation strategy for the detected circles to understand whether they belong to a depot rooftop or not. Datasets used from GeoEye-1 (0.5m GSD) and Google Earth (0.5m GSD).

To separate oil tank regions from the background, X. Cai et al [4] proposed a new visual EMHC saliency model. Here, mathematical morphology based on multi-scale histogram contrast, Hough transform and SVM classifier were used. For oil tank detection Hough transform was used and to confirm the oil tank SVM was used. It failed to detect dark oil tanks. Datasets are taken from large-field and high-resolution remote sensing images are downloaded from Google was used.

M. S. Islam [5] used wavelet (new threshold function) and Hough transform (HT) for multiple moving targets detection and this method is inefficient to detect targets more accurately.

A. O. Ok et al [6] used positively and negatively affected orientation \& magnitude images for all pixels in image at single radius method. But 
validation strategy is not given to understand whether detected circle belongs to a tank rooftop or not. Datasets are tested on panchromatic GeoEye-1 images $(50 \mathrm{~cm}, 11$ bits).

P. Viola et al [7] used an extension of the rectangle filters from Viola and Jones to the motion domain. But this method is not efficient in cluttered backgrounds under difficult illumination.

Z. Shi et al [8] proposed model as histograms of oriented gradients (HOGs) to generate a hypothesis using AdaBoost algorithm, hyper spectral algorithm. It was failed when dealing with ships near land, this approach will give poor performance. There are some issues present to dealing these facts. Datasets are collected from Google earth having the sizes of these images range from $3000 \times 3000$ to $5000 \times$ 5000 , and their resolutions are all $1 \mathrm{~m}$ was used.

\section{Proposed methodology}

The block diagram of our proposed methodology is shown in Fig.1. The steps involved in the proposed method are as follows:

- Pre-processing

- Candidate detection

- Feature extraction

- Classification

\subsection{Pre-processing}

The Otsu's thresholding method is used for preprocessing the images. Pre-processing is often considered to be the first step in image analysis. In general, pre-processing is one of the most difficult tasks in digital image processing. The Otsu's thresholding pre-process method chooses the threshold to minimize the intra class variance of the thresholded black and white pixels i.e., thresholding is used to extract an object from its background by assigning an intensity value $\mathrm{T}$ (threshold) for each pixel such that each pixel is either classified as an object point or a background point. The purpose is to subdivide an image into meaningful nonoverlapping regions, which would be used for further analysis. The Eq.(1) defines variance of classes to separate it.

The weighted within-class variance is:

$$
\sigma_{w}^{2}(t)=q_{1}(t) \sigma_{1}^{2}(t)+q_{2}(t) \sigma_{2}^{2}(t)
$$

Weights $q_{1}$ and $q_{2}$ are the probabilities of the two classes separated by a threshold and $\sigma_{1}^{2}$ and $\sigma_{2}^{2}$ are variances of these two classes.

Where the class probabilities are estimated as:

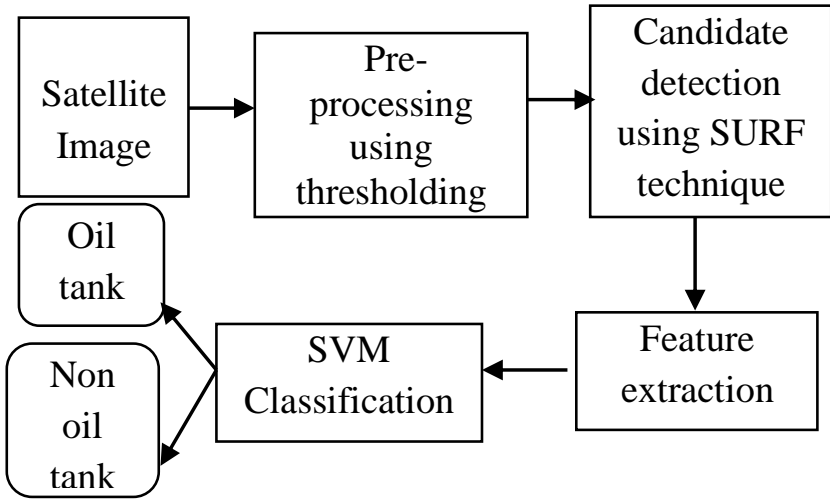

Figure.1 Block diagram of proposed method

$$
q_{1}(t)=\sum_{i=1}^{t} P(i) \quad q_{2}(t)=\sum_{i=t+1}^{I} P(i)
$$

And the class means are given by:

$$
\mu_{1}(t)=\sum_{i=1}^{t} \frac{i P(i)}{q_{1}(t)} \quad \mu_{2}(t)=\sum_{i=t+1}^{I} \frac{i P(i)}{q_{2}(t)}
$$

Finally, the individual class variances are:

$$
\begin{aligned}
\sigma_{1}^{2}(t) & =\sum_{i=1}^{t}\left[i-\mu_{1}(t)\right]^{2} \frac{P(i)}{q_{1}(t)} \\
\sigma_{1}^{2}(t) & =\sum_{i=1}^{t}\left[i-\mu_{1}(t)\right]^{2} \frac{P(i)}{q_{1}(t)}
\end{aligned}
$$

\subsection{Candidate detection}

The SURF technique is used to find the candidate (oil tank) detection from a pre-processed image. Here, initially calculates intensity of pixels present in the pre-processed images and original images, and also calculates interest points in the preprocessed images original images. It is calculated by SURF technique. Further, the pre-processed images and original images are compared based on interest points and intensity value calculated. With the help matched points, it detects that part from original image which matches interest point in pre-processed image. Thus, in this way candidate detection is done. SURF uses square-shaped filters as an approximation of Gaussian smoothing. Filtering the image with a square is much faster if the integral image is used:

$$
S(x, y)=\sum_{i=0}^{x} \sum_{j=0}^{y} I(i, j)
$$


The sum of the original image within a rectangle can be evaluated quickly using the integral image, requiring evaluations at the rectangle's four corners. SURF uses a hessian based blob detector to find interest points. The determinant of a hessian matrix expresses the extent of the response and is an expression of the local change around the area.

$$
H(x, \sigma)=\left[\begin{array}{ll}
L_{x x}(x, \sigma) & L_{x y}(x, \sigma) \\
L_{x y}(x, \sigma) & L_{y y}(x, \sigma)
\end{array}\right]
$$

Where,

$$
\begin{aligned}
& L_{x x}(x, \sigma)=I(x) \frac{\partial^{2}}{\partial x^{2}} g(\sigma) \\
& L_{x y}(x, \sigma)=I(x) \frac{\partial^{2}}{\partial x y} g(\sigma)
\end{aligned}
$$

$L_{x x}(x, \sigma)$ in Eq.(8) is the convolution of the image with the second derivative of the Gaussian. The heart of the SURF detection is non-maximalsuppression of the determinants of the hessian matrices.

The purpose of a descriptor is to provide a unique and robust description of a feature a descriptor can be generated based on the area surrounding a interest point. The SURF descriptor is based on Haar wavelet responses and can be calculated efficiently with integral images. It need to determine the orientation. By determining a unique orientation for interest point, it is possible to achieve rotational invariance. Before the descriptor is calculated the interest area surrounding the interest point are rotated to its direction.

The SURF algorithm does add another detail to speed up matching, which is the sign of Laplacian.

$$
\nabla 2 L=\operatorname{tr}(H)=L_{x x}(x, \sigma)+L_{y y}(x, \sigma)
$$

The laplacian is the trace of the hessian matrix Eq.(10) and when calculating the determinant of the hessian matrix these values are available. It is a matter of storing the sign. The reason to store the sign of the Laplacian is that distinguishes between bright blobs on dark backgrounds and vice versa. It is only necessary to compare the full descriptor vectors if it has the same sign, which can lower the computational cost of matching.

\subsection{Feature extraction}

The feature extraction is play is major role in the classification. In this paper, we have used well known features such as Histogram Oriented
Gradient (HOG), Gray Level Co-occurrence matrix (GLCM), Completed Local Binary Pattern (CLBP), Centre Symmetric Local Binary Pattern (CSLBP), unique Unsorted vec for classification. These features are further used for classification using SVM.

The HOG is a feature descriptor used in computer vision and image processing for the purpose of object detection. HOG descriptor focuses mainly on silhouette contours. The technique counts occurrences of gradient orientation in localized portions of an image. The HOG gradients descriptor is that local object appearance and shape within an image can be described by the distribution of intensity gradients or edge directions. The image is divided into small connected regions called cells, and for the pixels within each cell, a histogram of gradient directions is compiled. The descriptor is the concatenation of these histograms. The HOG parameters were adopted after a set of experiments performed with the SVM classifier. The HOG window size is fixed as the bounding box size, the block overlap is set at half of the block size and the histogram channel is set as 9 .

GLCM is simple texture descriptor which works well for highly textured images.

A CLBP is a type of visual descriptor used for classification in computer vision. It is simple and efficient texture descriptor. This function returns either a local binary pattern coded image or the local binary pattern histogram of an intensity. The CLBP function are computed using $\mathrm{N}$ sampling points on a circle of radius $\mathrm{R}$ and using mapping table defined by MAPPING in CLBP function. It is a powerful feature for classification.

CSLBP function takes patch or image as input and return Histogram of CSLBP operator. In this function, threshold is used as 0.1 .

Unique unsorted vec function returns the same data as in input, but with no repetitions and in original order. This function is just a layer over the excellent and well known Matlab 'unique' function. The unique function sorts the returned results which are not always desirable. So this function does the same, but the element order does not change. This function is highly similar to 'unique', but it operates on arrays only (numerical, character and cell arrays). It returns only the resulting array.

\subsection{Classification}

Support vector machine (SVM) is one of the best classifier. It analyzes the data and recognizes patterns with the associated learning algorithms and constructs a hyperplane for classification and that 
hyperplane only helps to classify into two categories. SVM maximizes the margin between the classes and minimize the classification error. In this work linear kernel function and polynomial kernel function is used. The linear kernel function is defined in Eq.(11) and polynomial kernel function is defined in Eq.(12).

$$
K\left(\mathbf{x}_{i}, \mathbf{x}_{j}\right)=\mathbf{x}_{i}^{T} \mathbf{x}_{j}
$$

Where, $K\left(x_{i}, x_{j}\right)$ is the kernel function and $x_{i}$ is the training data.

$$
K\left(\mathbf{x}_{i}, \mathbf{x}_{j}\right)=\left(1+\mathbf{x}_{i}^{T} \mathbf{x}_{j}\right)^{p}
$$

Where $x_{i}, x_{j}$ are vectors in the input space i,e. vectors of features computed from training samples.

\section{Experimental results and performance analysis}

\subsection{Datasets}

The proposed method is tested on datasets which is taken from SPOT-5 satellite images, QuickBird satellite images, GeoEye-1 satellite images and Google Earth images. Google Earth images consists industrial test site. Test is done on total 76 images taken from these satellite images. Among these 76 images, 48 images are taken from Google Earth, 9 images from GeoEye-1, 10 images from QuickBird satellite and remaining 9 images are from SPOT-5 satellite. Out of which, 40 images are of oil tanks and remaining 36 images of circular shaped on oil tanks like well images are there. The challenges in datasets are of having different environment, illumination, complex background and of noisy data preset in it. The sample input images are shown in Fig.2.

\subsection{Performance evaluation measures}

In this paper, for evaluating classification results the precision and recall measures are used. Precision measure is a measure of fidelity. This high Precision value indicates the low number of false positives detected $[9,10]$.

$$
\operatorname{Pr} \text { ecision }=\frac{\text { True Positive }}{\text { True Positive }+ \text { False Positive }}
$$

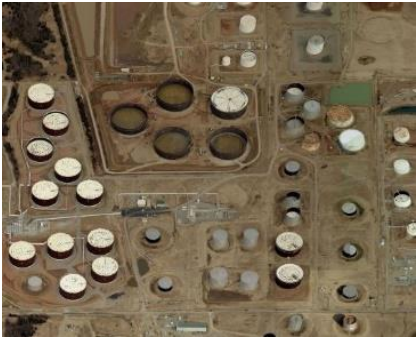

(a)

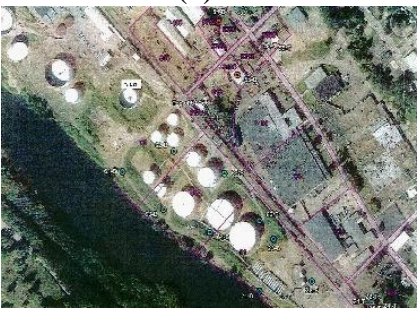

(c)

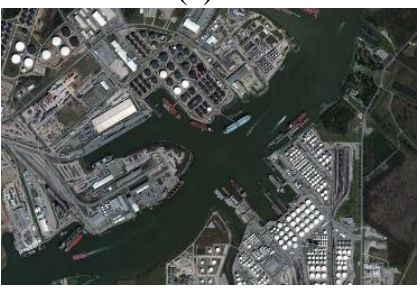

(e)

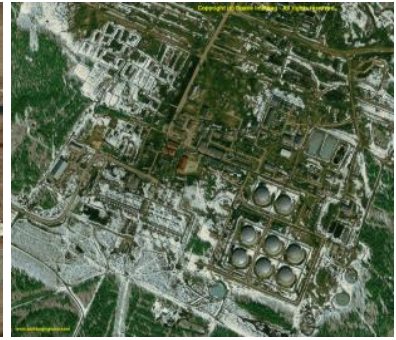

(b)

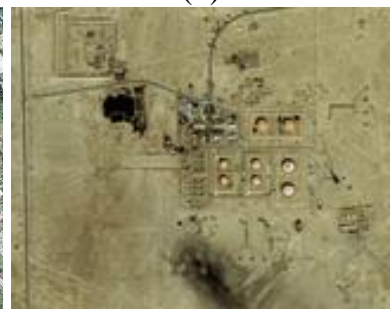

(d)

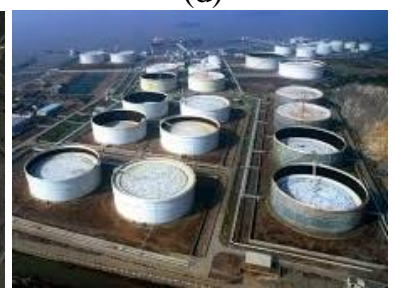

(f)
Figure.2 Sample input images: (a) Image from SPOT-5 satellite, (b) Image from QuickBird Satellite, (c) Google Earth Image, (d) GeoEye-1 Satellite image, (e) SPOT-5 satellite images, and (f) Google earth image

Recall is a measure of completeness. The high recall value is indicates the high detection rate.

$$
\text { Re call }=\frac{\text { True Positive }}{\text { True Positive }+ \text { False Negative }}
$$

\subsection{Experimental results}

The experimental results are presented in two parts. In the first part the candidate detection results are analyzed; and in the second part, the results of the feature-based classification method are analyzed. We have used the precision and recall measures to determine the performance of the results.

\subsubsection{Candidate detection}

In this paper, we have used SURF technique to detect candidate part from the whole image. In this part, initially images are pre-processed into objects using Otsu's threshold method then pre-processed image is compared with the original image for detect the oil tank using SURF technique. The sample Candidate (oil tank) detection result is given in Figs.3, 4, 5 and 6. In Fig.3 (a) is original image and Fig.3 (b) is its corresponding segmented object using threshold method. In Fig.4 (a) shows the 
SURF feature as Strongest 300 points as feature extraction of original image and Fig.4 (b) shows the SURF feature as Strongest 300 points as feature extraction of segmented object. In Fig.5 shows matching points between original image and segmented object with the help SURF technique. In Figs.6 (a), (b), and (c) shows the detection of oil tanks present in the original image.

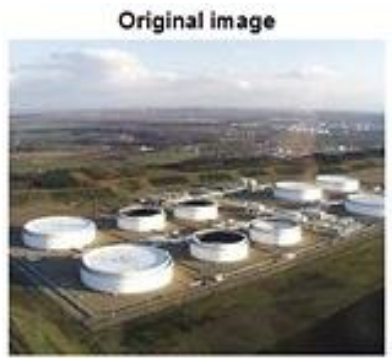

(a)

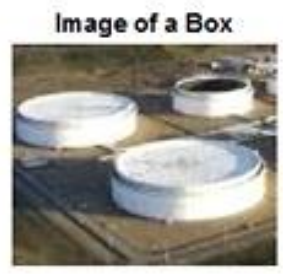

(b)
Figure.3 (a) original image and (b) segmented object

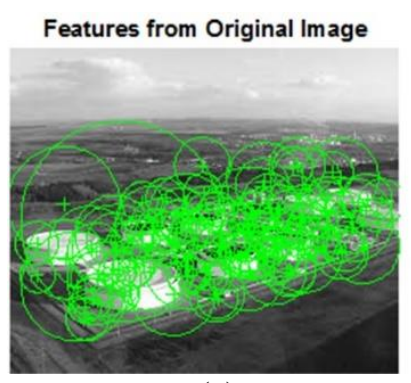

(a)

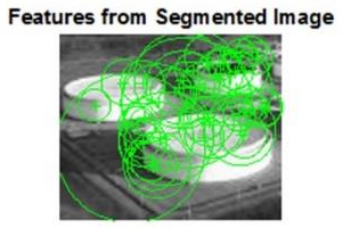

(b)
Figure.4 (a) Strongest 300 Points as features from Original and (b) Strongest 300 Points as features from segmented objects

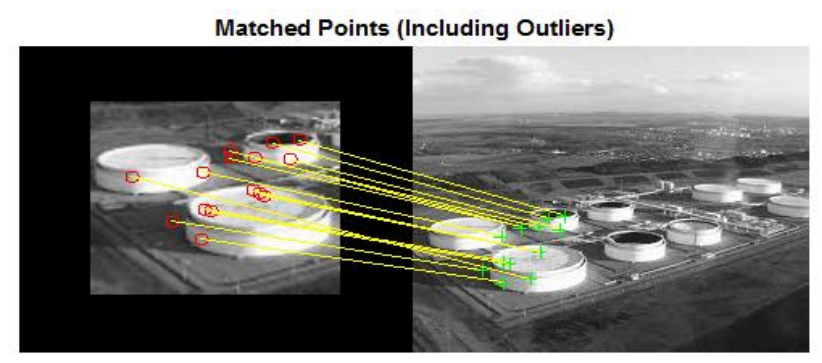

Figure.5 Matching points from original image and segmented object

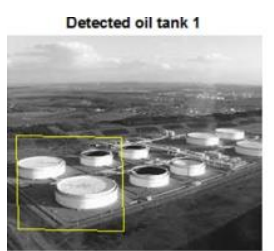

(a)

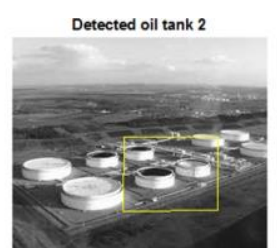

(b)



(c)
Figure.6 Detection of oil tanks present in original image: (a) $\operatorname{tank} 1$, (b) tank2, and (c) tank3

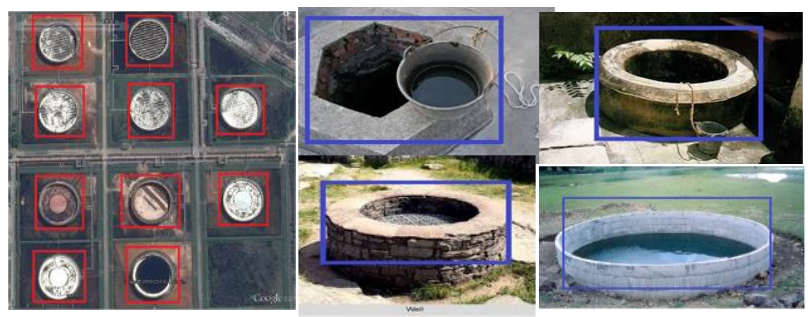

Figure.7 oil tanks are marked in red colour and non oil tanks are marked in blue colour

Table.1 Statistical analysis of input images with different feature based methods showing false positive, false negative, precision, recall for Linear Kernel of SVM

\begin{tabular}{|c|c|c|c|c|}
\hline Methods & $\begin{array}{c}\text { False } \\
\text { positive }\end{array}$ & $\begin{array}{c}\text { False } \\
\text { negative }\end{array}$ & $\begin{array}{c}\text { Precision } \\
(\%)\end{array}$ & $\begin{array}{c}\text { Recall } \\
(\%)\end{array}$ \\
\hline HOG & 2 & 3 & 95 & 92.68 \\
\hline GLCM & 18 & 2 & 55 & 91.66 \\
\hline CLBP & 0 & 0 & 100 & 100 \\
\hline CSLBP & 4 & 4 & 90 & 90 \\
\hline $\begin{array}{c}\text { Unique } \\
\text { Unsorted } \\
\text { vec }\end{array}$ & 0 & 0 & 100 & 100 \\
\hline
\end{tabular}

Table.2 Statistical analysis of input images with different feature based methods showing false positive, false negative, precision, recall for polynomial kernel of SVM

\begin{tabular}{|c|c|c|c|c|}
\hline Methods & $\begin{array}{c}\text { False } \\
\text { positive }\end{array}$ & $\begin{array}{c}\text { False } \\
\text { negative }\end{array}$ & $\begin{array}{c}\text { Precision } \\
(\%)\end{array}$ & $\begin{array}{c}\text { Recall } \\
(\%)\end{array}$ \\
\hline HOG & 0 & 0 & 100 & 100 \\
\hline GLCM & 12 & 1 & 70 & 96.55 \\
\hline CLBP & 0 & 0 & 100 & 100 \\
\hline CSLBP & 1 & 0 & 97.5 & 100 \\
\hline $\begin{array}{c}\text { Unique } \\
\text { Unsorted } \\
\text { vec }\end{array}$ & 1 & 0 & 97.5 & 100 \\
\hline
\end{tabular}

\subsection{Classification result}

In this paper, SVM classification is used to classify the images into oil tank and non oil tank images. Here, for the classification linear kernel and polynomial kernel function is used. For training total 76 images with 40 oil tanks and 36 non oil tanks were used. To test candidate detection result as oil tanks and non oil tanks the popular features are HOG, GLCM, CLBP, CSLBP and unique Unsorted vec are used. Table.1 shows statistical (false positive, false negative, precision and recall) analysis of input images with different feature based methods for linear kernel of SVM. The HOG features earned the $95 \%$ precision rate and $92.68 \%$ recall rate. The GLCM features method obtained the $55 \%$ precision rate and $91.66 \%$ recall rate. The CLBP features method obtained the $100 \%$ precision rate and $100 \%$ recall rate. The CSLBP features attained 90\% precision rate and $90 \%$ recall rate. Unique unsorted vec features secured the $100 \%$ precision rate and 
$100 \%$ recall rate for linear kernel function. From the results, it is observed that the CLBP and unique unsorted vec feature methods are provided high detection rate than other methods in linear kernel.

Similarly, Table. 2 shows statistical (false positive, false negative, precision and recall) analysis of input images with different feature based methods for polynomial kernel of SVM. The HOG features earned the $100 \%$ precision rate and $100 \%$ recall rate. The GLCM features method obtained the $70 \%$ precision rate and $96.55 \%$ recall rate. The CLBP features method obtained the $100 \%$ precision rate and $100 \%$ recall rate. The CSLBP features attained $97.5 \%$ precision rate and $100 \%$ recall rate. Unique unsorted vec features secured the $97.5 \%$ precision rate and $100 \%$ recall rate for polynomial kernel function. From the results, it is observed that the CLBP and HOG feature methods are high detection rate than other methods in polynomial kernel. Further, Table. 1 and Table. 2 are shown graphically in Figs. 8 and 9 for easy interpretation values.

In addition, the subjective comparison of classification result is shown in Fig.7. The true oil tanks are marked in red colour and non oil tanks are marked in blue colour. From these results it analysed that CLBP feature method is out performed than other methods.

Furthermore, the analysis on false positive and false negative rate for the different feature based methods for linear kernel function is shown in Fig.10. It is observed that CLBP and unique unsorted vec don't have any false positive and false negative. Similarly, analysis on false positive and false negative rate for the different feature based methods for polynomial kernel function is shown in Fig.11. Here observed that HOG and CLBP doesn't have any false positive and false negative.

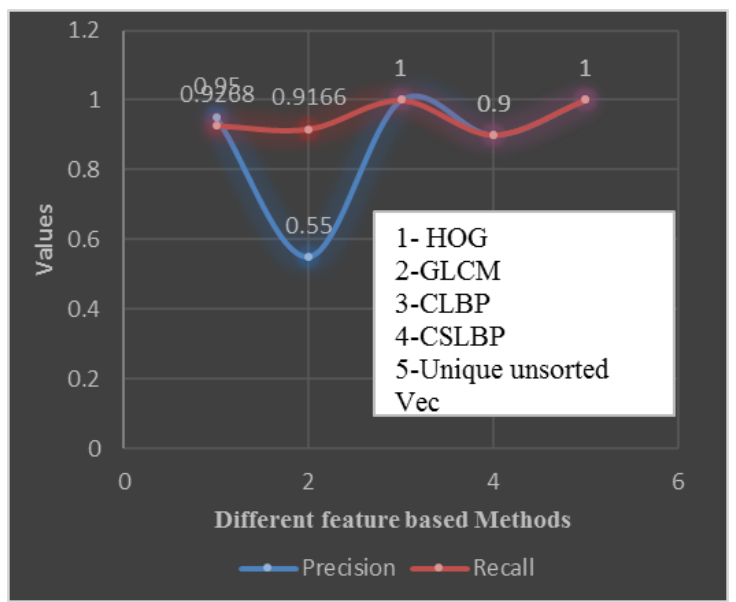

Figure.8 Comparison of precision \& recall values of different feature based methods using SVM linear kernel of classification results

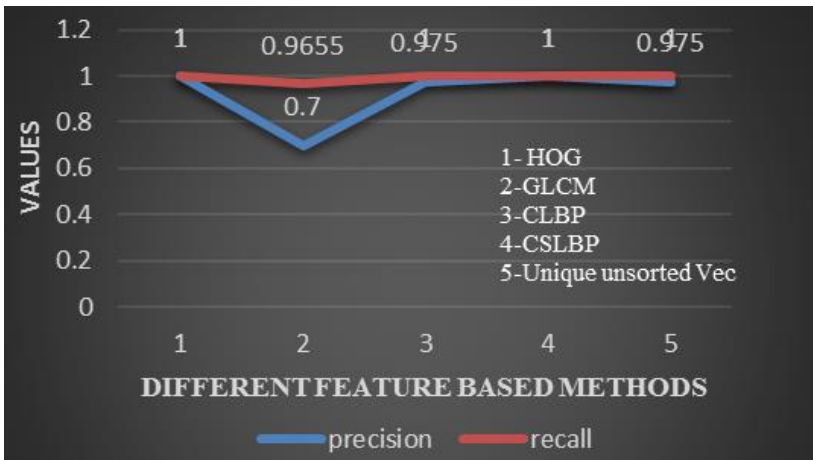

Figure.9 Comparison of precision \& recall values of different feature based methods using SVM polynomial kernel of classification results

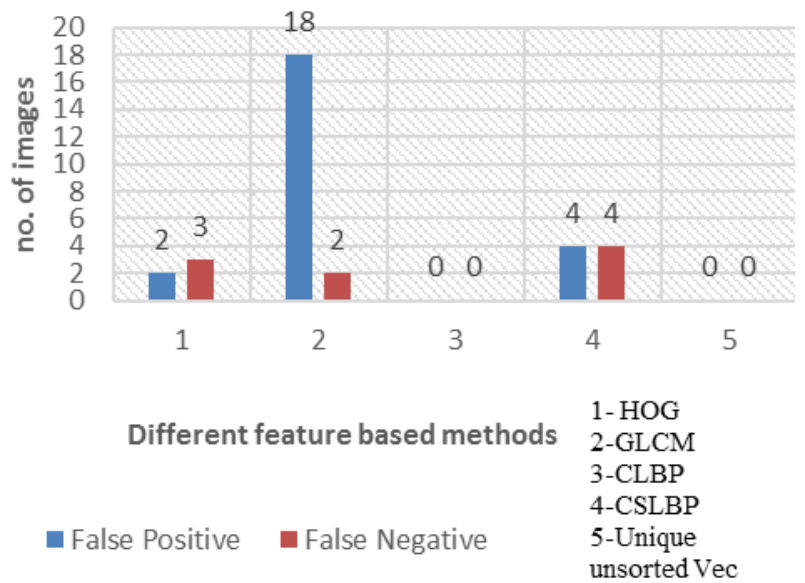

Figure.10 Comparison of analysing false positive $\&$ false negative of different feature based methods using SVM linear kernel of classification results

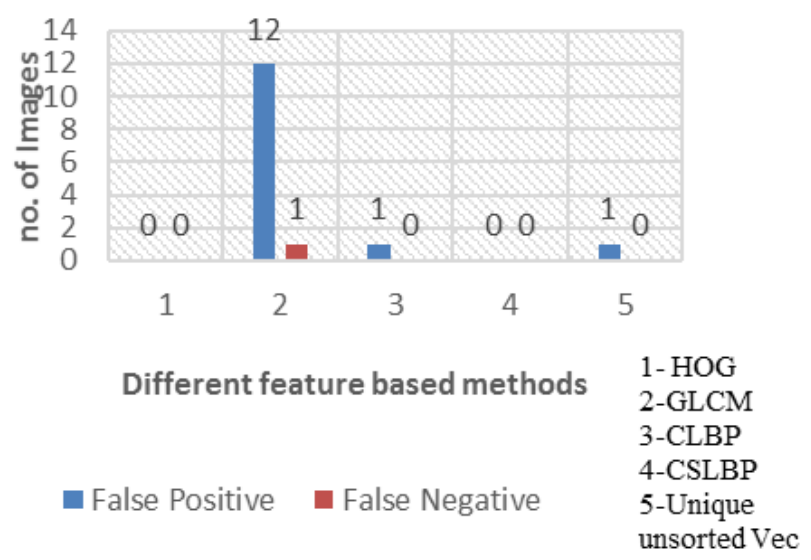

Figure.11 Comparison of analysing false positive $\&$ false negative of different feature based methods using SVM polynomial kernel of classification results

\section{Conclusion}

In this paper, an enhancement of algorithm is proposed for oil tank detection using SURF technique and SVM classifier. In this method, initially pre-processing is done using Otsu's threshold method. Secondly, SURF technique is applied on pre-processed image for candidate 
detection. Next, features are extracted from candidate detection image for classification. Finally, the candidate detection images are classified into oil tank and non oil tank using SVM classifier. The experimental result proved that the SURF technique using CLBP feature indicates high detection rate than other feature based methods.

In future we are interested to incorporate new feature for efficient classification to assist the military and civilian for disaster monitoring in the real world.

\section{References}

[1] L. Zung, Z. Shi, and J. Wu, "A Hierarchical Oil Tank Detector With Deep Surrounding Features for High-Resolution Optical Satellite Imagery", IEEE Journal of Selected Topics in Applied Earth Observations and Remote Sensing, Vol.8, No.10, pp.4895-4909, 2015.

[2] N. Dalal, and B. Triggs, "Histograms of oriented gradients for human detection", In: Proc. of International Conf. on Computer Vision and Pattern Recognition, San Diego, CA, pp.1-8, 2005.

[3] A. O. Ok and E. Baseski, "Automated detection of oil depots from high resolution images: A new perspective", ISPRS Annals of the Photogrammetry, Remote Sensing and Spatial Information Sciences, Vol.2, No.3, pp.149, 2015.

[4] X. Cai, H. Sui, R.Lv, and Z. Song, "Automatic circular oil tank detection in high-resolution optical image based on visual saliency and Hough transform", In: Proc. of IEEE Workshop on Electronics, Computer and Applications, pp.408-411, 2014.

[5] M. S. Islam, J. Lee, K. Hwang, and U. Chong, "Development of moving target detection based on image processing techniques", In: Proc. of Science and Information Conference, pp.734738, 2015.

[6] A. O. Ok and E. Baseski, "Circular oil tank detection from panchromatic satellite images: a new automated approach", IEEE Geoscience and Remote Sensing Letters, Vol.12, No.6, pp.1347-1351, 2015.

[7] P. Viola, M. J. Jones, and D. Snow, "Detecting pedestrians using patterns of motion and appearance", International Journal of Computer Vision, Vol.63, No.2, pp.153-161, 2005.

[8] Z. Shi, X. Yu, Z. Jiang, and B. Li, "Ship detection in high-resolution optical imagery based on anomaly detector and local shape feature", IEEE Transactions on Geoscience and Remote Sensing, Vol.52, No.8, pp.4511-4523, 2014.

[9] R. Soundrapandiyan, and P.V.S.S.R. Chandra Mouli, "A novel and robust rotation and scale invariant structuring elements based descriptor for pedestrian classification in infrared images", Infrared Physics \& Technology, Vol.78, pp.1323, 2016.

[10] R. Soundrapandiyan, and P.V.S.S.R. Chandra Mouli, "Adaptive pedestrian detection in infrared images using fuzzy enhancement and top-hat transform", International Journal of Computational Vision and Robotics, Vol.7, No.1/2, pp.49-67, 2017. 